\title{
Inversion of Toeplitz Band Matrices
}

\author{
By William F. Trench
}

\begin{abstract}
An algorithm for inverting Toeplitz matrices is simplified for Toeplitz band matrices. In some cases, the simplification yields formulas for the elements in the first row and column of the inverse, from which the remaining elements can be easily calculated. Two examples are given. In any case, the simplification yields a recursive method for computing the first row and column of the inverse of an $n$th order Toeplitz band matrix with $O(n)$ operations, where $O\left(n^{2}\right)$ are required with the more general algorithm.
\end{abstract}

1. Introduction. A Toeplitz matrix is of the form

$$
T_{n}=\left[\begin{array}{lllll}
\phi_{0} & \phi_{-1} & \phi_{-2} & \cdots & \phi_{-n} \\
\phi_{1} & \phi_{0} & \phi_{-1} & \cdots & \phi_{-n+1} \\
\phi_{n} & \phi_{n-1} & \phi_{n-2} & \cdots & \phi_{0}
\end{array}\right]=\left[\phi_{r-s}\right]_{r, s=0}^{n} .
$$

In [5], the author derived an algorithm for inverting a hermitian Toeplitz matrix $T_{n}$ with $O\left(n^{2}\right)$ operations (rather than $O\left(n^{3}\right)$, as required by standard matrix inversion methods) and stated a similar algorithm for the nonhermitian case. These algorithms require that all principal minors of $T_{n}$ be nonzero. Zohar [8] derived the extended algorithm in detail, and Akaike [1] generalized Zohar's derivation to block Toeplitz matrices. Bareiss [2] gave an algorithm for solving the linear system $T_{n} x=y$ with $O\left(n^{2}\right)$ operations.

In this paper, we simplify the more general algorithm of [5] for the case where $T_{n}$ is a Toeplitz band matrix, by which we mean that there are integers $p$ and $q$, $1 \leqslant p, q<n$, such that

$$
\phi_{p} \neq 0, \phi_{-q} \neq 0, \phi_{r}=0 \text { if } r>p \text { or } r<-q .
$$

In some cases, including two recently treated by quite different methods [3], [4], this simplification yields formulas for the elements of the first row and column of $T_{n}^{-1}$, from which its remaining elements can easily be calculated by Theorem 1 , below. In any case, the simplification yields the first row and column of $T_{n}^{-1}$ with $O(n)$ operations, where $O\left(n^{2}\right)$ operations are required for the more general procedure of [5].

Received June 20, 1973.

AMS (MOS) subject classifications (1970). Primary 65F30; Secondary 15A57.

Key words and phrases. Toeplitz matrix, band matrix, inversion. 
2. Computation of $T_{n}^{-1}$ from its First Row and Column. We follow the notation of [5]; therefore, in applying the results given here, the reader should note that $T_{n}$ is of order $n+1$. If $T_{n-1}$ is nonsingular, we define $\left\{\psi_{0, n-1}, \cdots, \psi_{n-1, n-1}\right\}$ and $\left\{\eta_{0, n-1}, \cdots, \eta_{n-1, n-1}\right\}$ by

$$
\sum_{s=0}^{n-1} \phi_{r-s} \psi_{s, n-1}=\phi_{r+1}, \quad 0 \leqslant r \leqslant n-1 \text {, }
$$

and

$$
\sum_{s=0}^{n-1} \phi_{s-r} \eta_{s, n-1}=\phi_{-r-1}, \quad 0 \leqslant r \leqslant n-1 .
$$

We state the following theorem for reference below.

THEOREM 1. If $T_{n}$ and $T_{n-1}$ are both nonsingular, then $T_{n}^{-1}=\left[b_{r s n}\right]_{r, s=0}^{n}$ is given by

$$
\begin{array}{ll}
b_{00 n}=\Delta_{n-1}^{-1} ; & \\
b_{r 0 n}=-\Delta_{n-1}^{-1} \psi_{r-1, n-1}, & 1 \leqslant r \leqslant n ; \\
b_{0 s n}=-\Delta_{n-1}^{-1} \eta_{s-1, n-1}, & 1 \leqslant s \leqslant n ;
\end{array}
$$

and

$$
\begin{array}{r}
b_{r s n}=b_{r-1, s-1, n}+\Delta_{n-1}^{-1}\left(\psi_{r-1, n-1} \eta_{s-1, n-1}-\eta_{n-r, n-1} \psi_{n-s, n-1}\right), \\
1 \leqslant r, s \leqslant n,
\end{array}
$$

where

$$
\Delta_{n-1}=\phi_{0}-\sum_{s=0}^{n-1} \phi_{s+1} \eta_{s, n-1}
$$

or, equivalently,

$$
\Delta_{n-1}=\phi_{0}-\sum_{s=0}^{n-1} \phi_{-s-1} \psi_{s, n-1} .
$$

Equations (5)-(8) are stated in [5], but derived there only for hermitian $T_{n}$. The derivation for the general case appears in [8]. Equations (9) and (10) do not appear in [5] or [8], but the special case to which they both reduce when $T_{n}$ is hermitian is derived in [5]; the same proof yields (9) and (10).

3. The Difference Equations. Theorem 1 says that if $T_{n}$ and $T_{n-1}$ are nonsingular, then $T_{n}^{-1}$ can easily be calculated from the solutions of (3) and (4). The following theorem shows that if $T_{n}$ is a band matrix, these solutions can be found by solving two difference equations of order $p+q$.

THEOREM 2. If $T_{n-1}$ is nonsingular and $\left\{\phi_{j}\right\}$ satisfies (2), then $\left\{\psi_{j, n-1}\right\}_{j=0}^{n-1}$ can be extended to all integers $j$ as the solution of the difference equation

$$
\sum_{i=-q}^{p} \phi_{i} \psi_{r-i, n-1}=0, \quad-\infty<r<\infty
$$

that satisfies the conditions 


$$
\begin{array}{ll}
\psi_{j, n-1}=0, & n \leqslant j \leqslant n+q-1, \\
\psi_{j, n-1}=-\delta_{-1, j}, & -p \leqslant j \leqslant-1 .
\end{array}
$$

Similarly, $\left\{\eta_{j, n-1}\right\}_{j=0}^{n-1}$ can be extended to all $j$ as the solution of

$$
\sum_{i=-q}^{p} \phi_{i} \eta_{r+i, n-1}=0, \quad-\infty<r<\infty
$$

that satisfies

$$
\begin{array}{ll}
\eta_{j, n-1}=0, & n \leqslant j \leqslant n+p-1, \\
\eta_{j, n-1}=-\delta_{-1, j}, & -q \leqslant j \leqslant-1 .
\end{array}
$$

In terms of these extended sequences, (9) and (10) can be rewritten as

$$
\Delta_{n-1}=\phi_{-q} \eta_{-q-1, n-1}
$$

and

$$
\Delta_{n-1}=\phi_{p} \psi_{-p-1, n-1} .
$$

We omit the routine verification of this theorem. A special case, applicable to hermitian $T_{n}$, has been stated and used [6], [7] for finding minimum variance prediction formulas for finite moving-average processes.

Example 1. Hoskins and Ponzo [3] have given formulas for the inverses of Toeplitz band matrices with

$$
\phi_{j}=(-1)^{j}\left(\begin{array}{c}
k \\
m+j
\end{array}\right)
$$

in the case where $k=2 r$ and $m=r$. Theorems 1 and 2 yield $T_{n}^{-1}$ when $k$ and $m$ are any integers such that $1 \leqslant m \leqslant k-1$.

Let $p=k-m$ and $q=m$ in Theorem 2. With $\phi_{j}$ as defined by (16), the solutions of (11) and (13) are polynomials of degree not exceeding $k-1$. Applying (12) and (14) yields

$$
\psi_{j, n-1}=\frac{(-1)^{m-1}(j-n-m+1)_{m}(j+2)_{k-m-1}}{(n+1)_{m}(k-m-1) !}
$$

and

$$
\eta_{j, n-1}=\frac{(-1)^{k-m-1}(j-n-k+m+1)_{k-m}(j+2)_{m-1}}{(n+1)_{k-m}(m-1) !}
$$

where

$$
(x)_{0}=1, \quad(x)_{s}=x(x+1) \cdots(x+s-1), \quad s \geqslant 1 .
$$

Equation (15) yields

$$
\Delta_{n-1}=(n+m+1)_{k-m} /(n+1)_{k-m} .
$$

Given (17), (18) and (19), $T_{n}^{-1}$ can now be obtained from (5)-(8). 
Example 2. Rehnqvist [4] has given a method for inverting Toeplitz band matrices with

$$
\begin{aligned}
\phi_{j} & =1-|j| / m & & \text { if }|j|<m, \\
& =0 & & \text { if }|j| \geqslant m .
\end{aligned}
$$

If

$$
x_{r}=\frac{1}{\sqrt{m}} \sum_{j=1}^{m} y_{j+r}, \quad-\infty<r<\infty,
$$

where $\left\{y_{r}\right\}$ is a wide sense stationary time series with zero mean, unit variance and independent samples, then $E\left(x_{r} x_{r+j}\right)=\phi_{j}$; thus, $T_{n}$ is the autocovariance matrix of any $n+1$ successive samples of $\left\{x_{r}\right\}$.

Rehnqvist's procedure requires multiplying $T_{n}$ by matrices whose forms depend strongly on the residue class of $n$ modulo $m$. This can be avoided by using Theorems 1 and 2. If $\phi_{j}$ is given by (20), the characteristic polynomial of (11) is

$$
z^{m-1} \sum_{j=-m+1}^{m-1} \phi_{j} z^{j}=m^{-1}(1-z)^{-2}\left(1-z^{m}\right)^{2} ;
$$

thus

$$
\psi_{r, n-1}=\sum_{j=1}^{m-1}\left(B_{j, n-1}+r C_{j, n-1}\right) \exp (2 \pi i r j / m)
$$

where $\left\{B_{j, n-1}\right\}$ and $\left\{C_{j, n-1}\right\}$ are to be determined from (12). This problem is a special case of one solved in [6] in connection with linear minimum variance prediction of $x_{r+k}$ based on observed values of $x_{r}, x_{r-1}, \cdots, x_{r-n+1}$. Since $\sum_{j=0}^{n-1} \psi_{j, n-1} x_{r-j}$ is the best such estimate of $x_{r+1}$, the results of [6] apply here. The pertinent results follow.

Case 1. If $n \equiv 0(\bmod m)$, then

$$
\begin{array}{ll}
\psi_{j, n-1}=(n-j) /(n+1) & \text { if } j \equiv 0(\bmod m), \\
\psi_{j, n-1}=-(n-j) /(n+1) & \text { if } j \equiv-1(\bmod m), \\
\psi_{j, n-1}=0 & \text { otherwise; } \\
\Delta_{n-1}=(n+m) / m(n+1) . &
\end{array}
$$

Case 2. If $n \equiv 1(\bmod m)$, then

$$
\begin{array}{ll}
\psi_{j, n-1}=(n+m-j-2) /(n+m-1) & \text { if } j \equiv 0(\bmod m), \\
\psi_{j, n-1}=-(n+m-j-2) /(n+m-1) & \text { if } j \equiv-1(\bmod m), \\
\psi_{j, n-1}=0 & \text { otherwise; } \\
\Delta_{n-1}=(n+2 m-2) / m(n+m-1) . &
\end{array}
$$

Case 3. If $n \equiv k(\bmod m)$, where $2 \leqslant k \leqslant m-1$, then

$$
\psi_{j, n-1}=\frac{(n+2 m-2 k)(n+m-k-j)}{(n+m-k)(n+2 m-2 k+1)} \text { if } j \equiv 0(\bmod m),
$$




$$
\begin{aligned}
& \psi_{j, n-1}=\frac{-(m-k+j+1)}{(n+m-k)(n+2 m-2 k+1)} \quad \text { if } j \equiv k-1(\bmod m), \\
& \psi_{j, n-1}=-\frac{(n+m-k-j-1)}{(n+m-k)} \quad \text { if } j \equiv-1(\bmod m), \\
& \psi_{j, n-1}=0 \quad \text { otherwise; } \\
& \Delta_{n-1}=\frac{(n+2 m-2 k)(n+2 m-k)}{m(n+m-k)(n+2 m-2 k+1)} \text {. }
\end{aligned}
$$

Since $T_{n}$ is symmetric, $\eta_{r, n-1}=\psi_{r, n-1}$ and $T_{n}^{-1}$ can now be computed from (5)-(8).

4. The Modified Algorithm for $\left\{\psi_{r, n-1}\right\}$ and $\left\{\eta_{r, n-1}\right\}$. Even if the difference equations of Theorem 2 cannot conveniently be solved explicitly, they still provide a useful simplification in computing $T_{n}^{-1}$, as we now show.

The algorithm of [5] includes a recursive procedure for computing $\psi_{0, n-1}, \cdots$, $\psi_{n-1, n-1}$ and $\eta_{0, n-1}, \cdots, \eta_{n-1, n-1}$ with $O\left(n^{2}\right)$ operations, provided $T_{0}, \cdots$, $T_{n-1}$ are all nonsingular. For band matrices, this can be done with $O(n)$ operations.

THEOREM 3. Let $p+q \leqslant k<n-1$. If $T_{k}, T_{k+1}, \cdots, T_{n-1}$ are all nonsingular, then $\psi_{0, n-1}, \cdots, \psi_{n-1, n-1}$ and $\eta_{0, n-1}, \cdots, \eta_{n-1, n-1}$ can be computed as follows.

(a) Obtain $\psi_{0 k}, \cdots, \psi_{k k}$ and $\eta_{0 k}, \cdots, \eta_{k k}$ by solving (3) and (4) with $n-1=k$, and compute

$$
\Delta_{k}=\phi_{0}-\sum_{r=0}^{q-1} \phi_{-r-1} \psi_{r, k}
$$

or, equivalently,

$$
\Delta_{k}=\phi_{0}-\sum_{r=0}^{p-1} \phi_{r+1} \eta_{r, k}
$$

(b) For $k+1 \leqslant m \leqslant n-1$, compute

$$
\begin{aligned}
\psi_{m m} & =-\Delta_{m-1}^{-1} \sum_{s=m-p}^{m-1} \psi_{s, m-1} \phi_{m-s} \\
\eta_{m m} & =-\Delta_{m-1}^{-1} \sum_{s=m-q}^{m-1} \eta_{s, m-1} \phi_{-m+s} \\
\psi_{r m} & =\psi_{r, m-1}-\psi_{m m} \eta_{m-r-1, m-1}
\end{aligned}
$$

for $0 \leqslant r \leqslant q-1$ and $m-p+1 \leqslant r \leqslant m-1$;

$$
\eta_{r m}=\eta_{r, m-1}-\eta_{m m} \psi_{m-r-1, m-1}
$$

for $0 \leqslant r \leqslant p-1$ and $m-q+1 \leqslant r \leqslant m-1$; and $\Delta_{m}=\left(1-\psi_{m m} \eta_{m m}\right) \Delta_{m-1}$.

(c) Then compute 


$$
\psi_{r, n-1}=-\frac{1}{\phi_{-q}} \sum_{j=-p}^{q-1} \phi_{-j} \psi_{r-q+j, n-1}
$$

for $q \leqslant r \leqslant m-p$, with $\psi_{j, n-1}=-\delta_{-1, j}$ for $-p \leqslant j \leqslant-1$, and

$$
\eta_{r, n-1}=-\frac{1}{\phi_{p}} \sum_{j=-q}^{p-1} \phi_{j} \eta_{r-p+j, n-1}
$$

for $p \leqslant r \leqslant m-q$, with $\eta_{j, n-1}=-\delta_{-1, j}$ for $-q \leqslant j \leqslant-1$.

This theorem follows from Theorem 2 and recursion formulas given in [5] for $\left\{\psi_{r m}\right\}$ and $\left\{\eta_{r m}\right\}$. A special case of Theorem 3 for hermitian $T_{n}$ was stated in [7] in connection with minimum variance prediction of finite moving-average processes.

The essential difference between Theorem 3 and the general algorithm of [5] is that (23) and (24) are not available in the latter, and it is necessary to use (21) and (22) for $m$ values $(0 \leqslant r \leqslant m-1)$ each, rather than $p+q$.

5. Comments. In using Theorem 1 , it is worthwhile to note that $T_{n}^{-1}$ is symmetric about its secondary diagonal; i. e., $b_{n-s, n-r, n}=b_{r s n}$. If $\phi_{-j}=\bar{\phi}_{j}$, then $T_{n}^{-1}$ is also hermitian; moreover, the computations in Theorems 1,2 and 3 are, in this case, further shortened by the fact that $\psi_{r m}=\bar{\eta}_{r m}$.

Throughout this paper, we have excluded the case where $p=0$ or $q=0$, so that $T_{n}$ is triangular. Inversion of a nonsingular triangular Toeplitz matrix-band or not-is very simple: if

$$
\phi_{j}=0, \quad j<0,
$$

and (without loss of generality) $\phi_{0}=1$, then $T_{n}^{-1}$ is the Toeplitz matrix given by

$$
b_{r s n}=-\psi_{r-s-1} \text {, }
$$

with $\psi_{j}=0$ if $j<-1, \psi_{-1}=-1$, and

$$
\psi_{j}=\phi_{j+1}-\sum_{s=0}^{j-1} \phi_{j-s} \psi_{s}, \quad 0 \leqslant j \leqslant n-1 .
$$

To see this, note that (4) and (25) imply that $\eta_{s, n-1}=0$ and that (3) is a triangular system with solution (27). Now, (26) follows from (5)-(8).

Department of Mathematics

Drexel University

Philadelphia, Pennsylvania 19104

1. H. AKAIKE, "Block Toeplitz matrix inversion," SIAM J. Appl. Math., v. 24, 1973, pp. 234-241.

2. E. H. BAREISS, "Numerical solution of linear equations with Toeplitz and vector Toeplitz matrices," Numer. Math. v. 13, 1969, pp. 404-424. MR 40 \#8234.

3. W. D. HOSKINS \& P. J. PONZO, "Some properties of a class of band matrices," Math. Comp., v. 26, 1972, pp. 393-400. MR 46 \#2839. 
4. L. REHNQVIST, "Inversion of certain symmetric band matrices," Nordisk Tidskr. Informationsbehandling (BIT), v. 12, 1972, pp. 90-98. MR 46 \#10183.

5. W. F. TRENCH, "An algorithm for the inversion of finite Toeplitz matrices," J. Soc. Indust. Appl. Math., v. 12, 1964, pp. 515-522. MR 30 \#3891.

6. W. F. TRENCH, "On the extrapolation of a special class of stationary time series," Ann. Math. Statist., v. 36, 1965, pp. 1426-1432. MR 32 \#3244.

7. W. F. TRENCH, "Weighting coefficients for the prediction of stationary time series from the finite past," SIAM J. Appl. Math., v. 15, 1967, pp. 1502-1510. MR 37 \#1051.

8. S. ZOHAR, "Toeplitz matrix inversion: the algorithm of W. F. Trench," J. Assoc. Comput. Mach., v. 16, 1967, pp. 592-601. MR 40 \#1023. 